Running head: CULTURALLY DECENTERED PICTURES

Culturally De-Centered Test Design for Pictorial Reasoning Assessments

Joni M. Lakin

Associate Professor, Educational Studies

University of Alabama 


\begin{abstract}
Although methods have been developed for developing culturally "decentered" assessments that use languages, such as transadaptation strategies, pictures are often assumed to be culture-neutral and have not received similar attention. This paper describes CogAT test development in 20072011 on the adaptation of verbal and quantitative reasoning formats that relied exclusively on pictures for students in grades $\mathrm{K}$ to 2 . In the course of the test development, we applied crosscultural test adaptation practices and developed new resources for cultural fairness reviews that explicitly addressed cultural loading of pictures and concepts beyond the usual achievement domains. This paper describes these applications and provides resources for others seeking to develop culturally decentered, picture-based assessments.
\end{abstract}




\section{Culturally De-Centered Test Design for Pictorial Reasoning Assessments}

In the U.S., English language learner (ELL) students are a heterogeneous population of students that pose unique challenges to test development and administration. In response to the challenges, researchers have developed innovative and boundary-pushing solutions that expand our knowledge of assessment for ELL students as well as the general student population. In this paper, we present some of these innovations designed to enhance the validity and fairness of assessments for English language learners (ELLs) in the K-12 testing context. We present a set of tools we developed for improving the cultural fairness review process for standardized tests, specifically when the test is heavily reliant on pictures and everyday (not specifically academic) content.

The assessment context of this paper is a measure of cognitive reasoning abilities for students in K-12 schools: Cognitive Abilities Test Form 7 (CogAT 7). Reasoning skills are important predictors of academic success, but are distinguished from measures of achievement by focusing on common concepts while requiring examinees to find patterns and relatively novel insights about relationships between concepts (i.e., "going beyond the information given"). In contrast, achievement tests focus more on school-based learning and reflect past learning processes rather than eliciting new learning. Using verbal, quantitative, and figural concepts, ability measures tap into the overall efficiency of cognitive processes and strategies that enable students to learn new skills and solve problems.

Schools use ability tests like CogAT for a variety of purposes, including gifted/talented identification and differentiating instruction, often in conjunction with achievement data. Thus, there is the potential that ability tests can identify untapped potential among all students, raising the stakes for their cultural fairness. This is particularly true in districts with substantial numbers of English learners, which in the U.S. comprise mostly Spanish as the native language.

A serious concern in the assessment of cognitive abilities is whether measures of reasoning that include verbal and quantitative content allow all students to fully demonstrate their abilities. If the concepts are not equally familiar to all students, then performance is influenced more by prior learning and less by current reasoning processes. In previous forms of $\mathrm{CogAT}$, the tasks for young students (grades $\mathrm{K}$ to 2 ) in verbal and quantitative domains required students to listen to a teacher-read prompt and select the picture demonstrating the best answer to the question. These item formats required strong receptive language skills in English and resulted in undesirably strong correlations between the verbal and quantitative scores. 
To address these concerns, picture-based reasoning items tapping distinct verbal and quantitative concepts were developed for a paper-and-pencil assessment with no teacher-read prompts (only the introductory test directions are read aloud and can be readily translated to new languages; Lohman, 2012). The goal of this study was to explore the validity and fairness of picture-based measures of reasoning skills that do not requiring extensive receptive language comprehension.

Some researchers and test developers feel that presenting only pictorial or nonverbal content is enough to make a test "culture fair", free of or minimizing the role of culture in understanding the test content. However, Ortar (1972) expressed concern about the assumption that pictures or figures on nonverbal tests were universal. She argued that words are actually safer, because we are adept at checking their translatability by seeking the help of native speakers. Pictures are just assumed to translate and "any cultural differences are comparatively well hidden.” (p 120). In a similar vein, Semin (1972) described the confusion that a simple picture of a couple sitting in rocking chairs caused for a sample of children in Turkey. The chairs, the clothes, and the pastimes of the characters were all distinctly U.S. Midwestern (e.g., the older white woman in a work dress was knitting). All of the culture-specific features had to be modified in order for the children to easily recognize the picture and answer the question.

In our work, the non-translatability of pictures was a serious concern. Therefore, we developed the picture-based items with the same care that a language-based assessment requires to ensure greater cross-cultural validity. We approached this from the outset with the framework of of reducing cultural load (van de Vijver \& Poortinga, 1992) or "cultural decentering" a test (Geisinger \& McCormick, 2014) with respect to our target population of native English and Spanish speakers living in the U.S. However, resources for this type of test development were not available in the literature. Therefore, we adapted existing and developed new approaches to test development to create picture-based reasoning assessments.

\section{Our process}

Our test development process incorporated ideas and processes from the extensive literature on Universal Design (e.g., Johnstone, Thompson, Bottsford-Miller, \& Thurlow, 2008) and cross-cultural test adaptation (Hambleton et al., 2005; Solano-Flores \& Wang, 2015; Tanzer, 2005). Input from students, bilingual teachers, and professional translators were incorporated into the test development process. Six primary methods were used, which are described in the sections that follow. 
1. Concurrent development of items to create culturally decentered content

2. Decentered and back translations by professional translators

3. Labeling study to assess familiarity of illustrations

4. Bilingual teacher review and item elicitation

5. Additional fairness reviews with "cultural load" rating scale

\section{Concurrent development of prompts}

The usual practice of test development in the US is to create a test in English, then translate the test to Spanish and other necessary languages. This method is fraught with problems of translating the test, determining the quality of translations, finding a suitable sample of testtakers for the standardization, and deciding what kinds of interpretations can be made about test scores (Sireci, 2005). Tanzer (2005) is one of several researchers who have called for test developers to be proactive in developing multi-language test forms and to build forms simultaneously.

In some testing contexts, bilingual test developers can be recruited for the project. However, in small test development teams, this is not feasible. To bring in this expertise to our small monolingual test development team, we incorporated the support of colleagues with relevant cultural knowledge for items that would be less familiar to Hispanic students in the U.S. These team members included a native Spanish speaker from Venezuela, a native Spanish speaker from Southern California, and another colleague who grew up speaking Spanish with her originally Puerto Rican family.

These language experts, who were current or former K-12 teachers, reviewed newly drafted items created by the primary test development team and evaluated how familiar various concepts would be to students from a similar background to them. They suggested substitutions that would be more familiar, and, for Sentence Completion items, debated which Spanish translations would be most familiar to U.S. based Spanish speakers. One notable challenge was the word for "kite". Each collaborator knew at least 2-3 words for kite, but none overlapped all three dialects! When possible, we chose the most neutral or common translation, but we gave precedence to Mexican dialects, because of the large number of Spanish speaking EL students from Mexico.

\section{Bilingual teacher review workshops}

We realized early in the test development process that decentering content could also be thought of as identifying "etic" concepts (Geisinger \& McCormick, 2014), a term that refers to 
concepts or ideas that are shared between two cultures. In contrast to etic concepts, there are "emic" concepts that are unique to one of the cultures and not shared with the other. Crosscultural test development theory allows for emic test content as long as it is equally balanced between concepts familiar to each represented culture.

With this idea in mind, we sought to identify content that might be more familiar or derive from the cultural experience of Spanish-speaking U.S. schoolchildren. Our tactic to identify such content focused on a pool of bilingual educators in our region. A local school offered a dual-language program where bilingual teachers worked with native English- and native Spanish-speaking students (primarily from newly arrived immigrant families) with the goal of teaching academic content while also building bilingual skills in all students.

We held a three-day workshop with our team of four bilingual teachers from this program, one curriculum professor, and one member of the test development team. We began by providing teachers with a basic introduction to the test, to test development, and to our goal of generating a test that was equally familiar and fair to native English and Spanish-speaking students in the U.S.

We began by reviewing items from our existing item development pool. When a problem was identified, teachers suggested replacement options or completely new items. We also dedicated substantial time to brainstorming content that would be uniquely familiar to their native Spanish-speaking students. Ultimately, we were not able to develop more than a few viable items from this perspective. As a group, we generated items around music, cultural events like rodeos, and festival topics. Most of these items were eventually discarded for being too specific, unfamiliar to other students, or somewhat stereotyping of Mexican-American culture. Working with more experienced test developers who had the cultural expertise of these students might be more successful in the future.

\section{Labeling study}

As Ortar warned for this type of work, assessing the familiarity of illustrations was more slippery. One method we developed was a "labeling" pilot study. Essentially, we asked a small sample of young students to label illustrations they viewed with the goal of verifying that the images were easily identified by young students. It was not important that they labeled them exactly as we intended, but that they generally understood what each picture showed (i.e., it was familiar enough that a child aged 5-8 would recognize it) and were not distracted by specific details or unfamiliar styles. 
Because of their age, children engaged in the task with a parent. The parent asked the child for their responses and typed them into the relevant box. Two examples are shown below. In the first example, the indicated picture showed a child coloring. We needed to make sure the children knew about coloring books and immediately recognized that activity.

Because we recruited some bilingual children, responses could be in English or Spanish. Originally, we planned to present single images at a time, but we felt that pictures were more recognizable and specific when placed in context with the other pictures, as they would be seen in the actual test. Due to time constraints, fewer than a dozen students participated, but we felt the method held substantial promise for this type of item development.
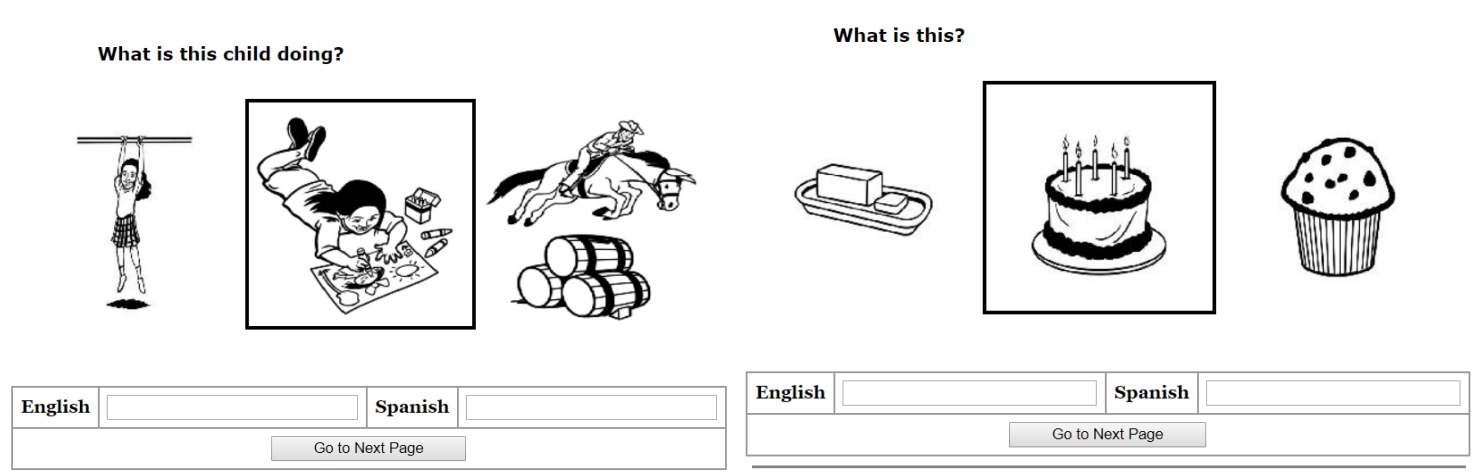

Figure 1. Labeling study design

To further assure that our pictures work for most US schoolchildren, we showed our pictures to half a dozen knowledgeable reviewers, all bilingual teachers of US school children with extensive experience with ELL students. They reviewed the pictures and noted which ones could be problematic for easy recognition by all test-takers. However, they found relatively few problems. We hope this is because we designed the test from the start with an eye towards translatability, but it may also reflect the difficulty of the task.

\section{Fairness reviews with Item Cultural Load Scale}

It is well known in the testing industry that fairness reviews often pick up idiosyncratic issues that rarely align with other indicators, such as DIF statistics or even other reviewers. We wanted to develop a more structured process that could increase the agreement of reviewers on the level of cultural fairness or cultural load of items.

Past fairness reviews of this measure simply asked reviewers to comment on any issues they perceived in items. We wanted to expand this process and share our perception of cultural load of assessments (based on theory and past research reviewed above) with reviewers so they might be more intentional about the way they reviewed the items for fair content. We hoped that this process might provide more clarity to the test development team of when items were 
problematic and what to do about the issues brought up. Some of the key areas we wanted reviewers to focus on included:

- Familiarity of content: Items should only require knowledge of content that is already familiar to U.S. students at the grade level(s) for which the items are intended.

- Familiarity of rules or classification schemes: Students may recognize the individual pictures in an item but be unfamiliar with the rule, category, or classification scheme needed to solve the item.

- Quality of art: The pictures should be drawn so that children can easily identify the concepts represented.

Two examples of culturally loaded items are provided in the Appendix that reflect level 3 or 4 cultural loads. An example of a level 2 cultural load is provided in Figure 2. Cultures vary particularly in what kinds of animals are considered part of a typical diet. This item assumes that smaller birds or songbirds would not be eaten, so the answer is E. This distracts from the focal concept of what "edible" means. Replacing the smaller bird with a clearly inedible item such as a toy would improve this item by removing a culturally loaded answer key. Another potential issue is whether the butter in a butter dish is equally familiar to all students. If they mistook it for soap or something else, it would unfairly attract them to choose that answer.

\section{Which of these is not edible?}
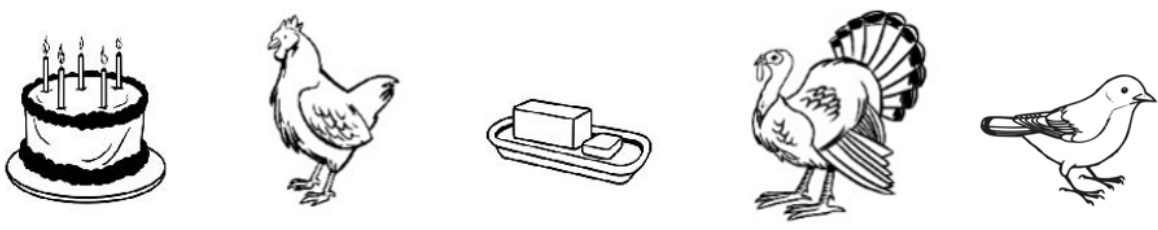

Figure 2. Example of level 2 cultural load.

In our fairness review, twenty-two reviewers from various backgrounds (including seven Hispanic school administrators, teachers, or school psychologists) participated. All instructions and the rating scale are provided in the Appendix. Each panel member provided ratings on each item (1-4). We flagged items for review when three or more reviewers rated the item at 3 or 4. All comments also considered and incorporated into item changes. Despite our best efforts, we still found very low agreement across raters with an intraclass correlation of just .19 (average measures, ICC type 2 [Two-way random effects]; Landers, 2011). On the one hand, each reviewer was basing ratings on their own perspective and reference group, so some disagreement 
is likely, but the low correlation of ratings is still notable. The average correlation between raters was just .08.

\section{How did it work?}

\begin{tabular}{|c|c|c|c|c|c|c|c|c|c|}
\hline & Zscore(VUSS) & \multicolumn{3}{|c|}{ Form 6} & \multicolumn{3}{|c|}{ Form 7} & \multirow[b]{2}{*}{ SD } & \multirow[b]{2}{*}{$\begin{array}{l}\text { Cohen's } \\
\text { d }\end{array}$} \\
\hline \multirow{4}{*}{ LEP } & LEVEL & $\begin{array}{l}\text { Typical } \\
\text { age }\end{array}$ & $\mathrm{N}$ & M & SD & $\mathrm{N}$ & $\mathrm{M}$ & & \\
\hline & K & $5 / 6$ & 1106 & -0.67 & 0.94 & 634 & -0.19 & 1.02 & 0.49 \\
\hline & 1 & 7 & 1143 & -0.73 & 1.05 & 99 & -0.31 & 0.84 & 0.44 \\
\hline & 2 & 8 & 1163 & -0.70 & 0.98 & 199 & -0.30 & 0.78 & 0.46 \\
\hline & 3 & 9 & 779 & -0.65 & 0.96 & 215 & -0.51 & 0.86 & 0.16 \\
\hline RL & K & & 5042 & -0.44 & 0.91 & 1922 & -0.20 & 0.95 & 0.26 \\
\hline & 1 & & 6359 & -0.42 & 0.94 & 447 & -0.17 & 0.93 & 0.27 \\
\hline & 2 & & 6187 & -0.46 & 0.94 & 1503 & -0.35 & 0.88 & 0.12 \\
\hline & A & & 5613 & -0.43 & 0.87 & 1351 & -0.42 & 0.86 & 0.02 \\
\hline & & & 1995 & -0.70 & 0.86 & 1945 & -0.18 & 0.98 & \\
\hline & & & & & & & & & 0.56 \\
\hline & & & 2135 & -0.71 & 0.94 & 430 & -0.23 & 0.88 & 0.52 \\
\hline & & & 2183 & -0.72 & 0.89 & 1204 & -0.15 & 1.01 & 0.61 \\
\hline & & & 2201 & -0.60 & 0.92 & 1205 & -0.37 & 0.87 & 0.26 \\
\hline
\end{tabular}

\section{Conclusions}

Although pictures may be assumed to be culturally universal, it is clear from the literature that pictures must be evaluated for cultural fairness with at least as much rigor as language. We presented strategies here that might be used by others in test construction to gauge the suitability of pictures using examinee and expert reviews. Some of the key points learned from this process included, first, the need to identify and understand the intended population(s) where the assessments will be used. Whether culture varies within a testing context (as in the U.S.) or when a test is transported between cultures, cultural variation needs to be understood at the outset of test development. Second, test developers should not assume that all illustrations are equal. While simple geometric shapes may (mostly) universally recognized, illustrations in particular must be assessed for the content and style of art to determine if all examinees will be equally familiar with the concept. We found in our work that detail and style of illustrations could vary substantially across artists and cultures. 
Finally, we found that the fairness review process still needs refinement and further study. Our proposed process is a qualitative improvement on the existing method, but did not lead to greater reviewer agreement. This begs a fundamental question, do we even seek agreement across reviewers? Or is it reasonable to expect differences given the diverse backgrounds of the people selected for sensitivity and fairness reviews? Better constraints and structure on the fairness review process, along with rigorous quantitative methods such as those reflected here, will help the field improve the fairness review process beyond a vague qualitative process to a more effective and structured approach to fairness review. What is absolutely clear is that pictures, just like words, must be subjected to fairness and cultural review in test construction. 


\section{References}

Geisinger, K. F. \& McCormick, C. (2012). Testing and assessment in cross-cultural psychology. In Naglieri, J., \& Graham, J. (Eds.), Handbook of Psychology (Vol. 10): Assessment Psychology (pp. 161-224). NY, NY: Wiley.

Johnstone, C. J., Thompson, S. J., Bottsford-Miller, N. A., \& Thurlow, M. L. (2008). Universal design and multimethod approaches to item review. Educational Measurement: Issues and Practice, 27(1), 25-36.

Landers, R. (2015). Computing Intraclass Correlations (ICC) as Estimates of Interrater Reliability in SPSS, The Winnower 2, doi: 10.15200/winn.143518.81744

Lohman, D. F. (2012). Cognitive Abilities Test (Form 7) Research Handbook. Itasca, IL: Riverside Publishing Company.

Lohman, D. F., Korb, K. A., \& Lakin, J. M. (2008). Identifying academically gifted English-language learners using nonverbal tests: A comparison of the Raven, NNAT, and CogAT. Gifted Child Quarterly, 52(4), 275-296.

Ortar, G. (1972). Some principles for adaptation of psychological tests. In L. J. Cronbach, \& P. J. D. Drenth (Eds.), Mental tests and cultural adaptation (pp. 111-120). The Hague: Mouton.

Semin (1972). Why certain tasks from mental tests must be adapted whereas operational tasks need not. In L.

J. Cronbach, \& P. J. D. Drenth (Eds.), Mental Tests and Cultural Adaptation (pp. 129-134). The Hague: Mouton.

Sireci, S.G. (2005). Using bilinguals to evaluate the comparability of different language versions of a test.

In R.K. Hambleton, P.F. Merenda, \& C.D. Spielberger (Eds.) Adapting educational and psychological tests for cross-cultural assessment (pp. 117-138). Mahwah, NJ: Erlbaum.

Solano-Flores, G., \& Wang, C. (2015). Complexity of Illustrations in PISA 2009 Science Items and Its Relationship to the Performance of Students from Shanghai-China, the United States, and Mexico. Teachers College Record, 117(1), 1-18.

Tanzer, N.K. (2005). Developing tests for use in multiple languages and cultures: A plea for simultaneous development. In R.K. Hambleton, P.F. Merenda, \& C.D. Spielberger (Eds.) Adapting educational and psychological tests for cross-cultural assessment (pp. 235-263). Mahwah, NJ: Erlbaum.

van de Vijver, F., \& Poortinga, Y. (1992). Testing in culturally heterogeneous populations: When are cultural loadings undesirable. European Journal of Psychological Assessment, 8(1), 17-24. 


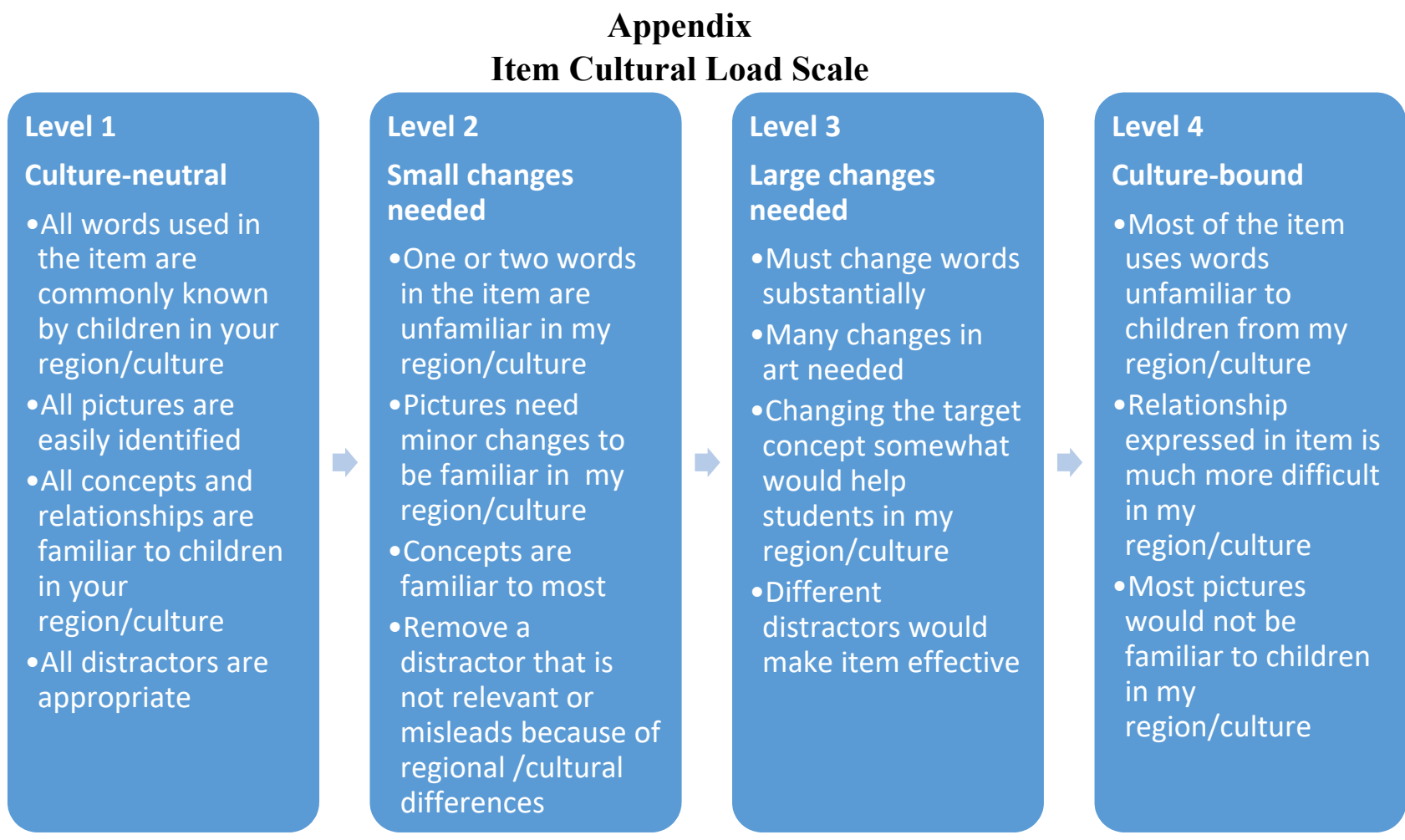

Here are a few things to consider as you classify the items according to the Item Cultural Load Scale.

1. Familiarity of content: Test items test reasoning abilities and should only require knowledge of content that is already familiar to U.S. students at the grade level(s) for which the items are intended.

a. Think about the concepts represented in the items - do students from your region or your culture know about these things? Would they encounter these concepts in books or on television?

b. For Levels $\mathrm{K} / 1$ through 3, if the content is familiar but the art is problematic, then tell us how to draw the pictures so the concepts would be more accessible. For example, what would the objects look like in your region?

c. For Levels 3 through 11/12, if you think an item uses words that are unfamiliar to students in your region or culture, please state the problem and let us know how we could make the wording more accessible.

2. Familiarity of rules or classification schemes needed to solve the item: Students may recognize the individual pictures in an item but be unfamiliar with the rule, category, or classification scheme needed to solve the item. If an item relies on conceptual relationships or categorizations that students in your region or culture don't usually use, and these relationships/categorizations are not taught in the schools, please let us know.

a. For example, are primary grade children in your region familiar with categorizing animals as farm vs. zoo animals?

b. If a rule or categorization would be unfamiliar to students in your region, please try to suggest ways to make it more familiar.

3. Quality of art: The pictures should be drawn so that children can easily identify the concepts represented. If you doubt that the children in your region/culture will be able to identify the concepts, please state the problem and try to suggest ways we can make the illustrations look more familiar.

4. Level 4 items: If an item will definitely not work for students in your region/culture, classify it as a Level 4 item on the Item Cultural Load Scale and tell us why it won't work. Then, try to think of a similar item that would be based on the everyday experiences of these students.

5. Items in Levels 2-3: Please suggest modifications that would make these items more culturally neutral. If possible, suggest alternative items that are similar in content but would appeal to the experiences of students from different parts of the country or different cultural backgrounds. 


\section{Obvious Example 1}

On this section of the test, students listen as the teacher reads a question in English and/or Spanish. Then, they mark the picture that answers the question.

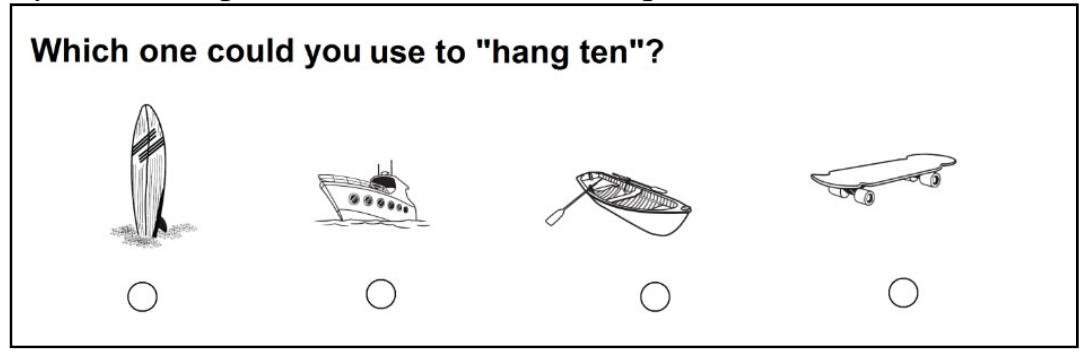

Target grade level: $\mathrm{K}$ to 3

On this test, reviewers should evaluate the sentence the teacher reads aloud, the pictures, and the concept in the item.

Do children from your region have enough exposure to the concept and/or wording of "hang ten" to reason with it as well as children from other regions? Students who live near the coasts probably have an advantage, so the concept should be changed. You could classify this item as Level 3 on the Item Cultural Load Scale, because it has substantial problems but they can likely be fixed. To make the item more accessible, you might suggest that the question ask "Which one is used to float on water but doesn't help you travel?" The surfboard, also, could be unfamiliar to students who do not live near an ocean. You might suggest that we replace it with something more recognizable that floats on water but doesn't help you travel, such as a life jacket.

\section{Obvious Example Item 2}

On this test, students must examine the pictures in the top row, decide how they are alike, and then identify the picture in the bottom row that belongs in the same group or category.

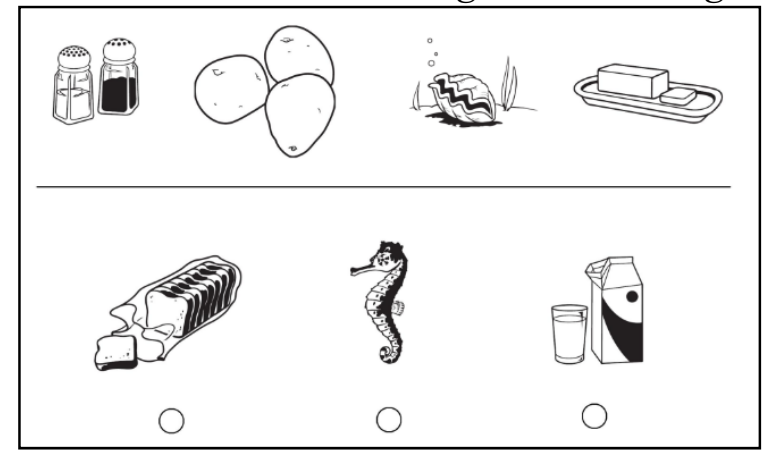

Target grade level: $\mathrm{K}$ to 3

First, consider the art. The illustrations should be drawn in a way that will be recognizable to students in your region/culture. Perhaps cardboard milk cartons are not common where you live, and children would not have seen them on television or in books or movies. If you come across an item like this, then identify the problem and tell us how we might correct it. If possible, provide a piece of art that shows how the milk container should look. Perhaps clams are not available in your part of the country, but children know what they look like from books and television. If this is the case, then the drawing of the clam would be acceptable. 
Next, consider the relationship represented. In the Picture Classification items, students must find a way to classify the pictures in the top row. In example 1, we chose something highly unusual-ingredients for making clam chowder.

Children who do not live in New England (and probably even those who do) may not be familiar with chowders in general or would not recognize the ingredients. We can't really fix this item without changing it entirely. We would flag the item as Level 4 (culture-bound). 